\title{
Ectopic expression of human acidic fibroblast growth factor 1 in the medicinal plant, Salvia miltiorrhiza, accelerates the healing of burn wounds
}

\author{
YaQing $\operatorname{Tan}^{1 \dagger}{ }^{1 \dagger}$ Kevin Yueju Wang ${ }^{2 \dagger}$, Nan Wang ${ }^{1}$, GangQiang Li ${ }^{1}$ and DeHu Liu ${ }^{1 *}$
}

\begin{abstract}
Background: Healing of burns is a complex process and very few effective treatments exist to facilitate the burn recovery process. Human acidic fibroblast growth factor 1 (FGF-1) plays an important role in a variety of biological processes, including angiogenesis, and tissue repair. Salvia miltiorrhiza is widely used in traditional Chinese medicine as an herb for the treatment of various diseases, including cardiovascular and cerebrovascular diseases, and traumatic injuries. We present that expression of FGF-1 in S. miltiorrhiza significantly accelerates the healing of burn wounds.

Results: The human fgf-1 gene was fused with a barley a-amylase signal peptide DNA sequence and driven by a $35 \mathrm{~S}$ promoter for constitutive expression in transgenic S. miltiorrhiza plants. The highest yield of recombinant FGF-1 obtained from leaves of transgenic S. miltiorrhiza lines was $272 \mathrm{ng} /$ fresh weight. Aqueous extracts from transgenic S. miltiorrhiza exhibited FGF-1 activity approximately 19.2-fold greater than that of the standard FGF-1. Compared to the standard FGF-1 or the extracts obtained from non-transgenic plants, it stimulated proliferation of Balb/c 3 T3 mouse fibroblast cells assessed with the standard MTT assay and promoted angiogenesis in the chicken embryo chorioallantoic membrane (CAM) assay. Topical application of the extract significantly accelerated the burn wound healing process.
\end{abstract}

Conclusions: The product appears to retain the biological activity of both FGF-1 as well as the medicinal properties of the plant. The extracts from transgenic S. miltiorrhiza combines the therapeutic functions of FGF-1 and the medicinal plant, S. miltiorrhiza. Topical application of the product can reduce the costs associated with extraction, purification, and recovery.

Keywords: Human acidic fibroblast growth factor 1, Medicinal plant, Salvia miltiorrhiza, Burn wound healing

\section{Background}

Various factors, such as heat, electricity, UV-light, and chemicals, can cause burns. Although most burns are not fatal, over 300,000 deaths occur globally each year as a direct result of burns, with $90 \%$ of burn deaths occurring in developing countries [1]. Healing of burns is a complex process and very few effective treatments exist to facilitate the burn recovery process. Antibiotics are often

\footnotetext{
*Correspondence: liudehu2006@126.com

${ }^{\dagger}$ Equal contributors

'Biotechnology Research Institute, Chinese Academy of Agricultural Sciences, 12 Southern Zhong Guan Cun Road, Beijing 100081, China

Full list of author information is available at the end of the article
}

used to prevent or treat infections and skin or synthetic grafts are used to facilitate the healing process in cases of third-degree burns [2].

Fibroblast growth factors (FGFs) comprise a large family genes involved in growth and differentiation. They are found in both invertebrates and vertebrates $[3,4]$. FGFs have been demonstrated to play essential functions in development, metabolism, and repair of various tissues and organs. Human FGFs contain 22 family members. Human FGF-1 is one of the most studied and characterized members of the superfamily. FGF-1 has various biological functions and has been associated with various 
stages of morphogenesis, tissue repair, angiogenesis and wound healing $[3,4]$.

FGF-1 is present in various tissues, especially within the extracellular matrix, however, isolating and purifying this protein is a complicated and tedious process. Recombinant DNA technology has enabled FGF-1 to be produced in E.coli [5], yeast [6], mammalian cells [7] and plants [8] for potential medical applications. Most studies designed to evaluate its effect on wound healing and tissue regeneration have administered recombinant FGF-1 directly to animal $[9,10]$ or human wound sites [10]. Like other FGFs, however, FGF-1 has low stability and is very sensitive to degradation by proteases $[3,4]$. Unfortunately, recombinant FGF-1 exhibits a very short half-life in vivo which limits its medical application.

Salvia miltiorrhiza, also known as Danshen, native to China and Japan, is a traditional Chinese medicinal (TCM) herb. The extract of its root has been widely used clinically to treat and prevent diseases, such as cardiovascular disease, hyperlipidemia, cancer, liver fibrosis and cirrhosis, etc. [11]. Leaf extract of S. miltiorrhiza also contains considerable amounts of pharmacological compounds even it is discarded as waste during root harvest. More than seventy compounds from $S$. miltiorrhiza have been investigated. Three major bioactive compounds, tanshinone-I, tanshinone-IIA, and cryptotanshinone have been extensively studied and reported to have a variety pharmacological activities, including anti-inflammatory [12], antioxidant [13], antitumor [14] and anti-platelet aggregation [15]. TCM compendiums indicate that S. miltiorrhiza reduces blood stasis by promoting blood circulation and repairing damaged tissues. The use of S. miltiorrhiza to treat coronary heart disease and hypertension, as well as for stroke patients, is in clinical phase IV trials (clinicaltrials.gov). However, like many TCM herbs, the precise mechanism underlying the biological activity of $S$. miltiorrhiza still remains to be completely elucidated.

In the present study, FGF-1 was constitutively expressed in the medicinal plant, S. miltiorrhiza. Presence and levels of FGF-1 were analyzed by ELISA and Western blot. Aqueous extracts of transgenic S. miltiorrhiza were applied directly to fertilized chicken egg embryo chorioallantoic membranes (CAM) and to second degree burn wounds on Sprague Dawley (SD) rats. Results indicated that the transgenic plants exhibited a both the biological function of FGF-1 and the medicinal properties of $S$. miltiorrhiza. Data indicated that the extract obtained from the transgenic plants could promote cell proliferation, speed up the growth of new blood vessels and significantly accelerate the burn wound healing process compared to the use of either FGF-1 or S. miltiorrhiza alone. The use of the transgenic plant extract also avoids the complicated process of FGF-1 extraction and purification.

\section{Results}

\section{Gene cloning and plant transformation}

Human FGF-1 was cloned into the expression vector, pBI121, with a Kozak consensus sequence inserted before the start codon and a barley alpha amylase signal sequence at the $5^{\prime}$ end, driven by a cauliflower mosaic virus 35S (CaMV35S) promoter (Figure 1) for strong constitutive expression. At least 120 independent putative transgenic events were obtained. All putative transformants were analyzed by PCR amplification using P1 and P2 primers. Results indicated that fifty-six of the putative transformants had the predicted $0.5 \mathrm{~kb}$ band.

\section{ELISA and western blot analysis}

The level of rFGF-1 produced in the confirmed transgenic lines was determined by ELISA. Results revealed that the yield of rFGF-1 in lines T65, T97 and T117 were131, 146 and $272 \mathrm{ng} / \mathrm{g}$ fresh leaf weight, respectively, as calculated by comparison with a standard curve constructed using commercial FGF-1 (see Additional file 1: Table S1).

Recombinant human acidic fibroblast growth factor 1 (rFGF-1) protein expression in transgenic lines T65, T97 and T117 was determined by the Western blot analysis (Figure 2). A positive response using FGF-1 monoclonal antibody demonstrated that transgenic lines (T65, T97, and T117) had a $16 \mathrm{kDa}$ band corresponding in size to standard FGF-1. No band was observed in protein samples extracted from WT plants. The results of the Western blot also indicated that T117 had a higher level of expression than T65. It corresponded with the results obtained in the ELISA assay. Since T117 had the highest level of rFGF-1, it was examined further. Transgene copy number in line T117 was determined by Southern blot analyses (see Additional file 2: Figure S1). Results indicated that there was a single T-DNA copy integrated in line T117.

\section{Proliferative effects of transgenic S. miltiorrhiza (T-SM) on cell proliferation in vitro}

After $72 \mathrm{~h}$ of culture, MTT assay revealed that wild-type S. miltiorrhiza (WT), standard FGF-1 (S-FGF) and T-SM

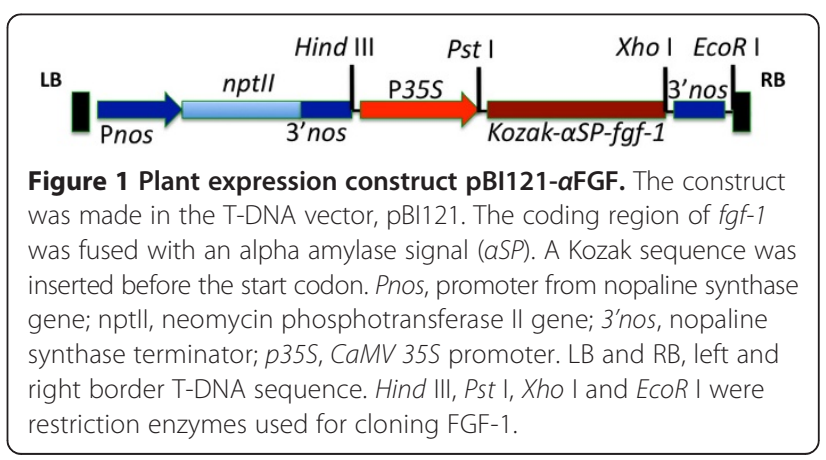




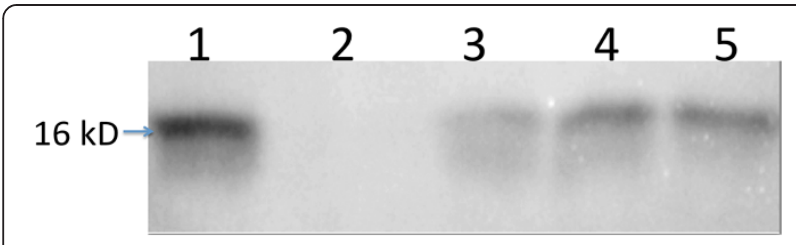

Figure 2 Western immunoblotting assay. Standard FGF-1 (Lane 1), rFGF-1 expression in wild type plants (Lane 2), and transgenic plant lines, T65 (Lane 3), T97 (Lane 4) and T117 (Lane 5). $10 \mu \mathrm{L}$ of supernatant from each sample was used in the immunoblotting assay.

significantly promoted Balb/c 3 T3 cell proliferation compared to the Saline control (Figure 3). The viability of the cells decreased when the cells were treated with diluted concentrations of samples. After the second ( $4^{2}$ fold $)$ dilution, WT showed had no effect on cellular viability. After the third $\left(4^{3}\right.$ fold) dilution, the absorbance of T-SM $(0.076 \pm 0.0034)$ was still significantly higher than that of S-FGF $(0.065 \pm 0.0026)$. The results indicated that treatment with T-SM greatly increased the cell proliferation compared with that of the other groups. The estimated effective dose (ED50) stimulating 50\% of the maximal cell number response was determined from MTT proliferation curves. The dilution fold of ED50 for S-FGF and T-SM were $4^{1.5}$ and $4^{1.47}$, respectively. The converted enzyme activity of T-SM $\left(4.8 \times 10^{6} \mathrm{IU} / \mathrm{mg}\right)$ was approximately 19.2 fold greater than that of the standard FGF- $1\left(2.5 \times 10^{5} \mathrm{IU} /\right.$ mg) (Table 1).

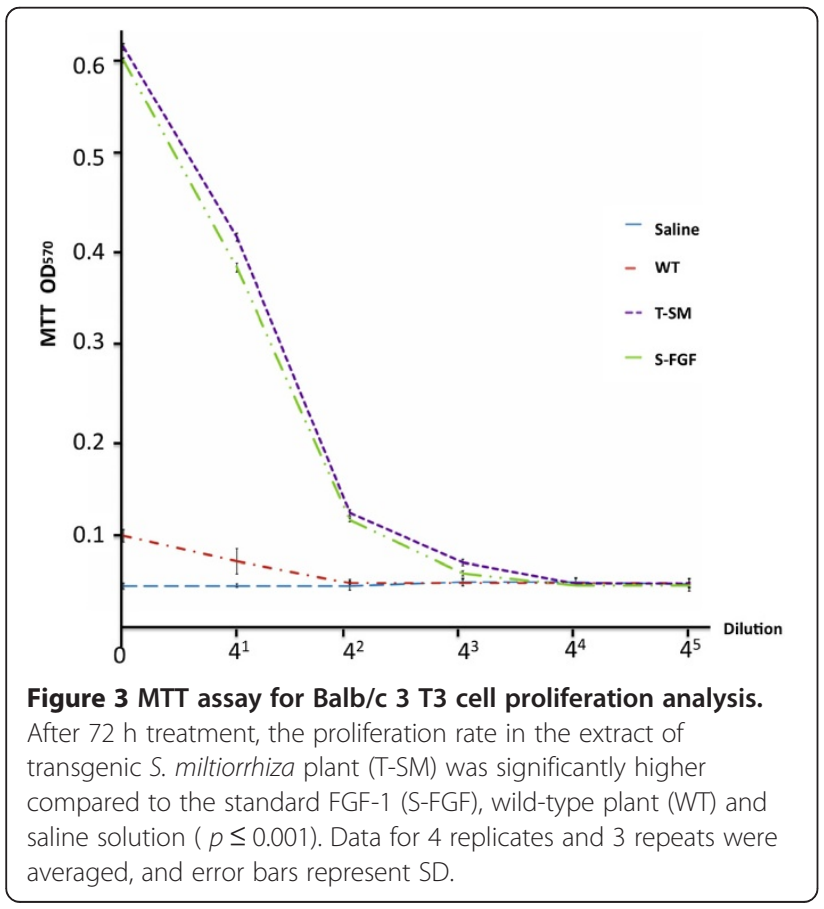

Table 1 Enzymatic activity of the recombinant FGF-1 from transgenic S. miltiorrhiza

\begin{tabular}{lllll}
\hline & FGF-1 content & ED50 (dilution) & AU/mL & U/mg \\
\hline S-FGF & $200 \mathrm{ng} / \mathrm{mL}$ & $4^{1.5}$ & 50 & $2.5 \times 10^{5}$ \\
T-SM & $10 \mathrm{ng} / \mathrm{mL}$ & $4^{1.47}$ & 48 & $4.8 \times 10^{6}$ \\
\hline
\end{tabular}

Effective dose 50 (ED50) is determined by MTT assay curve using Balb/c 3 T3 cells. The dilution fold is used to determine that compounds are active at that concentration to proliferate $50 \%$ of the cell growth. The enzymatic activity of recombinant FGF-1 from transgenic S. miltiorrhiza (T-SM) is determined in terms of $\mathrm{AU} / \mathrm{mL}$ in relation to a standard human acid FGF-1 (S-FGF) with a known activity $(50 \mathrm{AU} / \mathrm{mL})$. The specific enzymatic activity Units of FGF in T-SM was calculated as $48 \mathrm{AU} / \mathrm{mL}\left(4^{1.47} / 4^{1.50} \times 50 \mathrm{AU} / \mathrm{mL}\right)$, with a conversion of $(1000 \times 1000 / 10 \mathrm{ng}) \times 48=4.8 \times 10^{6} \mathrm{U} / \mathrm{mg}$

\section{T-SM stimulates angiogenesis in vivo}

The CAM assay is widely used to study angiogenesis in vivo. Our study demonstrated that treatment with extracts from WT, S-FGF and T-SM all stimulated angiogenesis (Figure 4). At day 11, a very few large vessels with only few branching were observed with in the samples treated with just saline. In comparison to the saline treatment, a greater number of large vessels and branches were formed in the WT treatment. T-SM treatment induced a significantly greater number of large vessels and branches compared to all of the other treatments. Even though the concentration of T-SM rFGF-1 used in the CAM assay was $1 / 18.5$ of the S-FGF treatment $(\approx 2.7 \mathrm{ng}$ vs. $50 \mathrm{ng}$, respectively), it stimulated more vessels. The greatest amount of branch formation was also observed with T-SM-induced angiogenesis in the CAM assay.

\section{T-SM Accelerates wound healing process}

Images of burn wounds treated with saline, WT, S-FGF (FGF-1 $1000 \mathrm{ng} /$ wound), and T-SM (rFGF-1 $\approx 2.7 \mathrm{ng} /$ wound) at $0,3,7,14$, and $21 \mathrm{~d}$ after administering the burn treatment are shown in Figure 5. On day 3, shrinkage of the wounds was observed in all groups. WT and saline groups had begun to form dark granulation tissue. In contrast, T-FGF-1 treated wounds appeared smooth and whitish. FGF-1 treated wounds were also smooth but exhibited some granulation tissue. On day 7, wounds in all groups continued to shrink and develop thick granulation tissue. On day 14, while considerable contraction of wounds was observed in all treatments, T-SM treated wounds began rebuilding healthy granulation tissue. On day 21, the S-FGF and T-SM treated wounds were almost healed, while WT and saline treated wounds were still very visible and whitish in appearance. Wound surface area tracing measurements (Figure 6) did not identify significant difference among the four treatments on day 0,7 and 14 and also revealed that the healing process appeared normal. On day 21, however, both T-SM and S-FGF treated wounds were significantly reduced $(\mathrm{P}<0.01)$ in wound surface area $(0.14 \pm 0.06$ and $0.21 \pm 0.05$, respectively) compared to the saline $(0.64 \pm 0.10)$ 


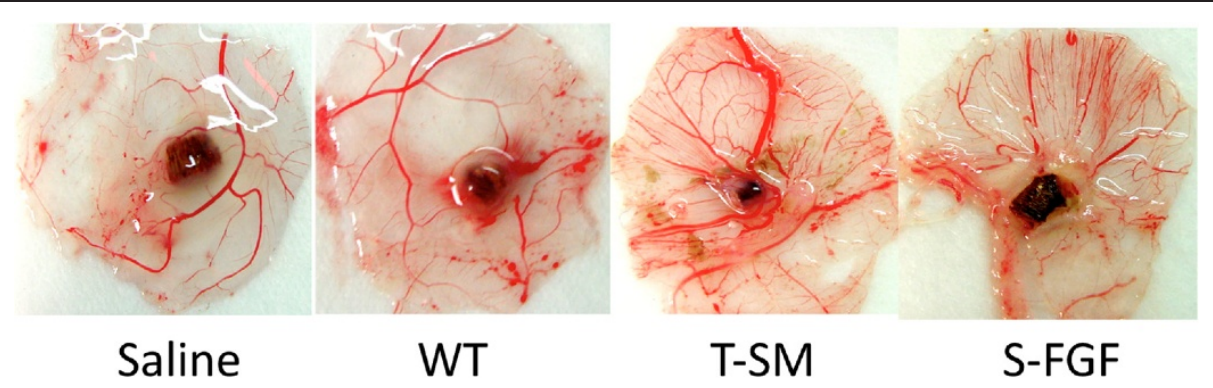

Figure 4 Extracts from transgenic plant promotes angiogenesis in the CAM assay. A: Saline solution was used as negative control. $5 \mu \mathrm{L}$ of aqueous extract from wild-type S. miltiorrhiza plants (WT) and transgenic S. miltiorrhiza (T-SM). S-FGF treatment contained 50 ng of FGF-1 produced in E. coli and obtained commercially.

and WT $(0.58 \pm 0.11)$ treated wounds. S-FGF and T-SM treated wounds were almost completely healed. No difference in wound surface area was observed between saline and WT treated burns. Importantly, while the concentration of FGF-1 in the S-FGF treatment was 1000 ng FGF-1/ wound, it was only $\approx 2.7 \mathrm{ng}$ rFGF-1 in the T-SM treatment. However, they exhibited a similar ability to accelerate the wound healing process.

\section{Histopathological assessments of burn wound healing process}

Histopathological analysis of normal tissues and the burn wound healing process was conducted by sectioning of tissues and staining with hematoxylin and eosin staining ( $\mathrm{H} \& \mathrm{E})$. A standard second degree burn wound was established (see Additional file 3: Figure S2). Normal skin had an intact cuticle, hair follicles, sebaceous glands, collagen and a prickle cell layer (A and B). Epidermal necrolysis was observed $24 \mathrm{~h}$ after burn treatment ( $\mathrm{C}$ and $\mathrm{D})$. In the dermis, dead cells disintegrated and collagen fibers became disorganized. Hair follicles were also damaged and vasodilation and congestion of the blood vessels were evident.

On day 7, the saline treated burn wounds exhibited inflammatory cells in epidermal necrolysis tissue. An inflammatory response in the wound area was not obvious
(Figure 7). In other treatments, homogeneous necrosis was evident in the epidermal exudate of burn wounds but with less inflammatory cells. The inflammatory response in the wound area was obvious with an increased number of inflammatory cells (B, C, D). On day 14, in contrast to saline treated wounds, the burn wounds in all other treatments exhibited rapidly growing granulation tissue, including fibroblasts, capillaries, and inflammatory cells. In S-FGF and T-SM treated burn wounds, a greater amount of newly formed epidermis began to migrate into the wound area (C and D). On day 21 , saline treated burn wounds still exhibited a thick surface layer of dead cells. A large number of inflammatory cells were also observed with some bleeding near the epidermis. An incomplete monolayer of epidermal cells was also observed. Burn wounds in all the other treatments exhibited good re-epithelialization. A thicker prickle cell layer was observed in S-FGF treated wounds than in TSM treated wounds. Tissue directly in the wound area began to form hair follicles and other skin appendages. T-SM treated wounds exhibited less necrotic tissue compared to all the other treatments.

\section{Discussion}

Our studies demonstrated that the medicinal plant, $S$. miltiorrhiza, can be used to produce functional human

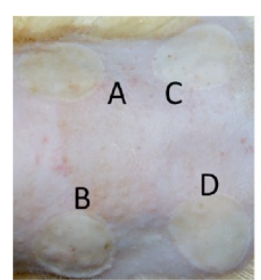

Day 0

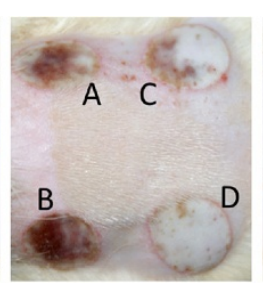

Day 3

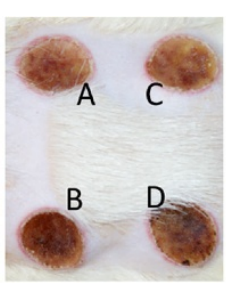

Day 7

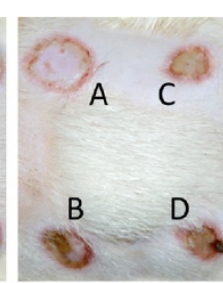

Day 14

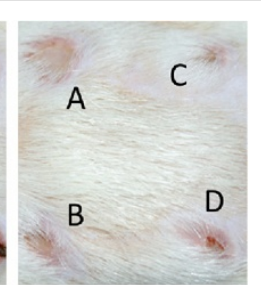

Day 21

Figure 5 Extracts from transgenic plants accelerate the burn wound healing process. Second degree burn wounds were administered on the dorsal skin of SD rats. The area of the burn wounds was treated with (A), $100 \mu \mathrm{L}$ of saline (B) aqueous extract from wild type plants; (C), $1000 \mathrm{ng}$ of commercial FGF-1, and; (D) $100 \mu \mathrm{L}$ of aqueous extract from transgenic S. miltiorrhiza. Photographs were taken on Day 0, 3, 7, 14 and 21 after burn injury was administered. 


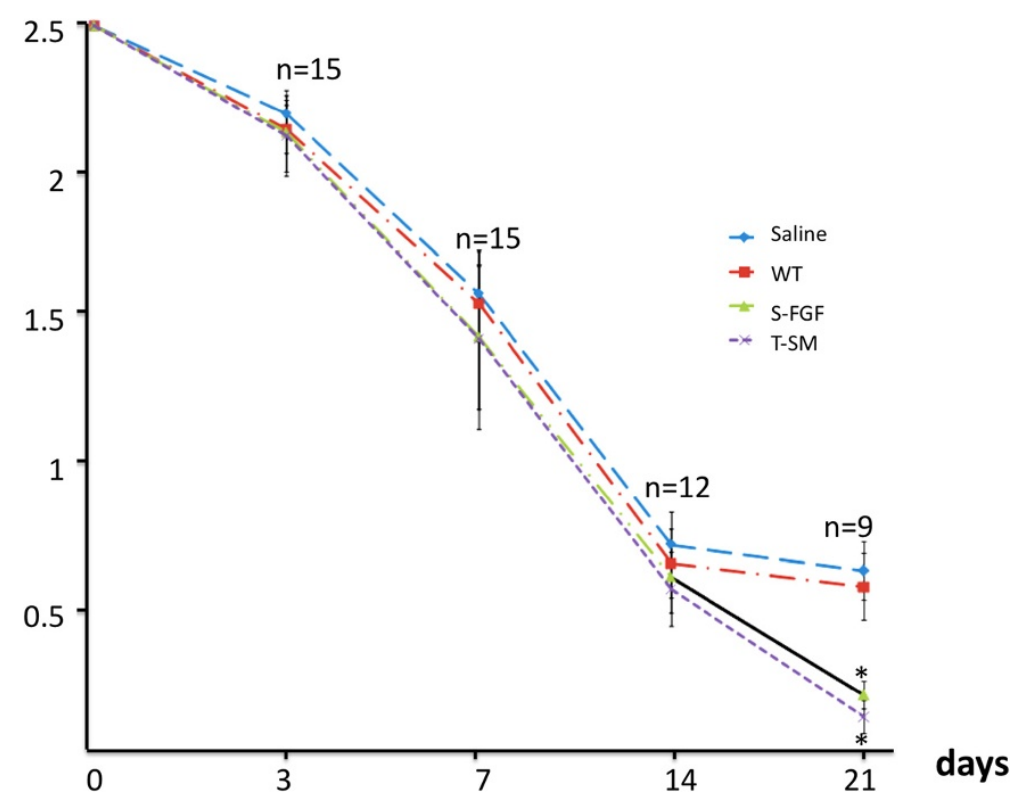

Figure 6 Burn wound surface assay. Area as evaluated using the transparent tracing method described by Bohannon and Pfaller (1983). Wounds were treated with either $100 \mu \mathrm{L}$ of saline, $1000 \mathrm{ng}$ commercial FGF-1, or $100 \mu \mathrm{L}$ aqueous extract from wild type or transgenic S. miltiorrhiza. Burn wound area was recorded on day $3(n=15), 7(n=15), 14(n=12)$ and $21(n=9)$. The data represent the Means \pm se. $n$ : rats number for each treatment. *indicates significant difference $(P<0.01)$ between the specified treatment and the saline treatment.

FGF-1. In addition to acquiring the therapeutic function of FGF-1, the transgenic plants also contained natural, endogeneous, medicinal compounds, such as tanshinone-I, tanshinone-IIA and cryptotanshinone. The therapeutic effect of the transgenic plants was significant and greater than using either FGF-1 or the medicinal plant alone. Western blot (Figure 2) assays indicated that FGF-1 was expressed in the transgenic $S$. miltiorrhiza.

The highest accumulation of recombinant FGF-1 level in transgenic S. miltiorrhiza was $272 \mathrm{ng} / \mathrm{g}$ fresh leave weight in line T117. We found that topical application of crude extracts from transgenic S. miltiorrhiza could enhance angiogenesis and accelerate the burn wound healing process.

The drawback in using plants to produce recombinant proteins is that plant cells often recognize the proteins as foreign and target them for degradation. Since only the expected single band was observed in the western blot assay, this indicated that the rFGF-1 in S. miltiorrhiza had not been subjected to proteolysis. Reports have suggested that foreign gene expression, and protein yield in transgenic plants could increase after producing several generations (T1-T4) of plants from self-pollinated primary (T0) transgenic lines $[16,17]$. We selected the line T117, which has a single transgene integration event as determined by Southern blot (Additional file 2: Figure S1), to produce subsequent generations in order to determine if the yield of rFG-1 in transgenic S. miltiorrhiza can be increased.

Extracts of non-transgenic S. miltiorrhiza can inhibit the stickiness of blood platelets and decrease fibrin $[11,15]$. The extract from this plant is widely used in
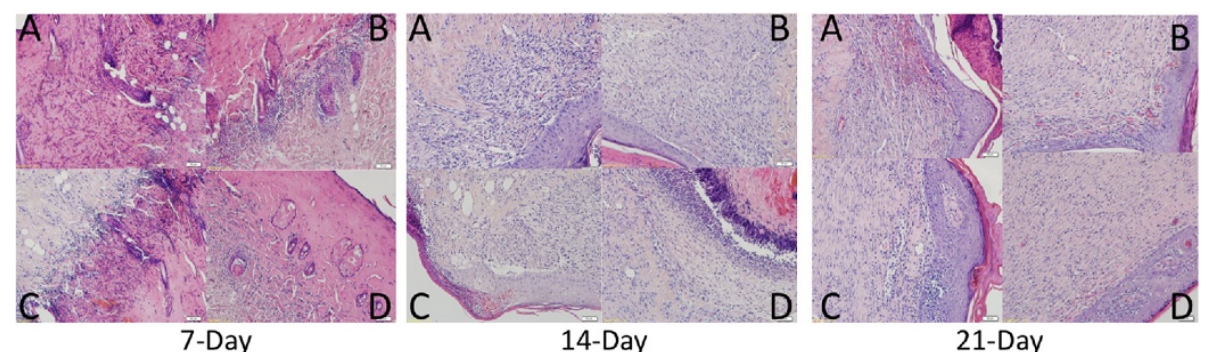

Figure 7 H\&E staining. Cryosections ( $2 \mu \mathrm{m}$ thick) stained with hematoxylin $(H)$ and eosin $(E)$ to examine the burn wound healing process after application of different treatments. Samples were taken on day 7, 14 and 21. A: $100 \mu \mathrm{L}$ of saline; B: $100 \mu \mathrm{L}$ saline aqueous extract from wild type (WT) S. miltiorrhiza; C: $1000 \mathrm{ng}$ FGF-1; and D: $100 \mu \mathrm{L}$ saline aqueous extract from transgenic S. miltiorrhiza. Micrographs were taken at 200x. 
traditional Chinese medicine to treat cardiovascular and cerebrovascular diseases [11]. One of its compounds, salvianolic acid, was reported to enhance in vitro angiogenesis in rat endothelial cells through the up-regulation of VEGF and VEGF receptors genes that promote cell growth and differentiation [18]. In the CAM assays, we also observed that the $S$. miltiorrhiza treatment produced more blood vessels (Figure 4). These results indicate that $S$. miltiorrhiza may facilitate angiogenesis. Very few studies have reported that $S$. miltiorrhiza has an effect on the burn wound healing process. One study indicated that injection of S. miltiorrhiza and ligustrazine can effectively reduce myocardial damage in patients with severe burns [19]. Animal studies also indicate that S. miltiorrhiza enhances the wound healing process $[20,21]$. Randomized clinical trial of S. miltiorrhiza suggested that it is effective in reducing skin flap ischaemia necrosis after mastectomy [20]. S. miltiorrhiza can stimulate the cell proliferation and it has the potential to be used for wounding healing or cosmetic treatment [22]. In this study, the $S$. miltiorrhiza treatment stimulated fibroblast cell proliferation (Figure 3). The burn wound healing process is complex. We did not observe that S. miltiorrhiza treatment of burn wounds reduced burn wound area compared to the saline treatment (Figures 5 and 6). H\&E staining, however, indicated that $S$. miltiorrhiza treatment can promote the formation of new cells and tissues to accelerate wound re-epithelialization. S. miltiorrhiza can promote the circulation system in wounded tissue (Figure 7). It may improve the burn wound healing process by removing necrotic cells, attenuating the inflammatory response and apoptosis [12,21], reducing oxidative stress [12], and possibly facilitate the repair of tubular epithelia structures [23].

The function of FGFs in tissue repair, angiogenesis and wound healing is well documented [3,4]. FGF-1 and FGF-2 promote cell proliferation and migration, as well as induce the physical organization of endothelia cells into blood vessel structures [24,25]. These properties can facilitate the burn wound healing process. The MTT, CAM assays, and burn wound healing test used in the present study also demonstrated that the application of FGF-1 enhanced cell proliferation and induced a greater number of new blood vessels and reduced the burn wound surface area more than treatments in which FGF-1 was absent (Figures 3, 4 and 5). These results were further supported by the histological analysis. The healing rate in the S-FGF treated wounds was more rapid compared to either the saline or WT treated wounds. The healing rate in S-FGF and T-SM treated burn wounds was similar. $\mathrm{H} \& \mathrm{E}$ staining revealed less necrosis in T-SM treated wounds than in S-FGF treated wounds. Approximately $2.7 \mathrm{ng}$ of the rFGF-1 was used in the T-SM treatment while 1000 ng FGF-1 was used in the S-FGF treatment.
This indicates that the T-SM treatment was significantly more efficient in its effect on angiogenesis and the burn wound healing process. The FGF-1 used in the S-FGF treatment was produced in E.coli. Common microbial hosts such as E. coli can produce high yields of recombinant protein but lack the requisite machinery for posttranslational modification required for protein stability and bioactivity [26]. In contrast, the protein synthesis process in plants is very similar to animal cells. The structure and function of rFGF-1 derived from transgenic $S$. miltiorrhiza may be more similar to native, human FGF-1 and thus have higher activity than FGF-1 obtained from $E$. coli. S. miltiorrhiza exhibits anti-inflammatory [12], antioxidant [13] and anti-bacterial activity [27]. The medicinal properties of $S$. miltiorrhiza combined with FGF-1 functions appears to accelerate burn wound healing by improving blood circulation and providing more nutrients and oxygen to the wound area.

FGFs are readily degradable in vivo, which results in a loss of biological activity and function [3,4,28,29]. FGF-1 biological activity was maintained in the extracts obtained from transgenic S. miltiorrhiza and applied topically to burn wounds. S. miltiorrhiza is a medicinal plant and contains natural compounds that also have various pharmaceutical functions. Overexpression of $f g f-1$ in $S$. miltiorrhiza provides the benefits of both FGF-1 and the medicinal properties of the plant, which may enhance its therapeutic effects. Plant specific glycans can harm humans and injection of recombinant plant therapeutic proteins may result in an immunogenic response in humans [30]. Topical application of transgenic $S$. miltiorrhiza extracts avoids plantspecific glycosylated immunogenic concerns and reduces the complex and costly purification and recovery process associated with the production of therapeutic compounds in microorganisms.

\section{Conclusions}

We successfully overexpressed $f g f-1$ in transgenic $S$. miltiorrhiza. Supernatant from homogenated transgenic $S$. miltiorrhiza plants promoted angiogenesis and accelerated the burn wound healing process. The product system combines the therapeutic functions of FGF-1 and the medicinal plant, S. miltiorrhiza. Topical application of the product can reduce the costs associated with extraction, purification, and recovery. The yield of rFGF-1 could be further increased by optimizing the gene codon, targeting the protein to the specific subcellular compartments, or selfing of high-expressing lines to obtain homozygous plants. The mechanism associated with the enhanced biological activity of S. miltiorrhiza and FGF-1 combined in the same extract will require further study. 


\section{Methods \\ Materials}

The plasmids, pUC-haFGF (containing $f g f-1$ gene), pT $\Omega 4 \mathrm{~A}$, the plant expression construct pBI121, and Agrobacterium tumefaciens LBA4404 are materials maintained in our lab. Seeds of S. miltiorrhiza were purchased from Shangluo Northwestern Medical Plant Company of Shaanxi (Shaanxi, China). Balb/c 3 T3 mouse fibroblast cells were bought from the Cell Culture Center, Institute of Basic Medical Sciences of Chinese Academy of Medical Sciences and School of Basic Medicine of Peking Union Medical College (Beijing, China). Fertilized eggs were obtained from the Institute of Animal Sciences (IAS), Chinese Academy of Agricultural Science (CAAS), Beijing, China. Male Sprague-Dawley (SD) rats (aged 6 to 7 weeks) were purchased from Vital River Laboratories Company (Beijing, China). All animal protocols were approved by the CAAS Institutional Ethics Committee.

\section{Plasmid construction}

In order to enhance recombinant FGF-1 expression level, a Kozak consensus sequence was inserted before the start codon and a barley alpha amylase signal peptide $[31,32]$ was fused to the FGF-1 sequence for extracellular targeting of the recombinant FGF-1. Three rounds of PCR were performed. The first round of PCR used P1 and P2 primers. P1 (forward): 5' -CTTTCTGCCAGCTT GGCCTCCGGACAAGTTTACAAGAAGCCAAAGTT GCTTTACT-3' (Italicized sequences indicate the partial 3 ' - end alpha amylase signal peptide sequence obtained from barely (Hordeum vulgare) and P2 (FGF-1 reverse); 5' -AACTCGAGTTAATCAGAAGAAACTGGCAAT-3' (Xho I site is italicized). PCR product resulting from the use of the P1 and P2 primers was used as the template for the second round PCR with P3 and P2 primers. P3 (forward):5' -TCCCTCTCCCTCTTCCTCGTCCTCCTT GGCCTTTCTGCCAGCTTGGCCTCCGGAC-3' (Italicized sequences indicate the middle region of the alpha amylase signal peptide sequence). The second round PCR product resulting from the use of the P3 and P2 primers was used as the template for the third round PCR with P4 and P2 primers. P4 (forward): 5' - AACTGCAG AACAATGGCGAACAAACATTTGTCCCTCTCCCTC TTCCTCGTCCTCC (Italicized sequences indicate the Pst I site and 5 '-end partial alpha amylase signal peptide sequence, respectively. The bold indicates the Kozak sequence). The third round PCR product was analyzed on a $1 \%$ agarose gel and subcloned into a TA cloning vector. After sequence verification (TaKaRa, Dalian, China), the product was digested using Pst $\mathrm{I} /$ Xho I and inserted between the $35 S$ promoter and Nos terminator of pT $\Omega 4 \mathrm{~A}$. The resulting $35 S$-(fgf-1)-Nos cassette was digested using Hind III/EcoR I and then inserted into a plant binary vector, pBI121 using the same sites, to generate the plant expression construct, pBI121-aFGF.

\section{Plant transformation and selection}

Transformation of S. miltiorrhiza utilized the method described by Horschet et al. [33] with modification. The binary vector pBI121-aFGF described above was introduced into A.tumefaciens strain LBA4404 using the triparental mating method. The resulting culture was plated on YEP (Yeast extract $10 \mathrm{~g} / \mathrm{L}$, Peptone $10 \mathrm{~g} / \mathrm{L}, \mathrm{NaCl} 5 \mathrm{~g} / \mathrm{L}$, $\mathrm{pH}$ 7.0) plates containing selective antibiotics (kanamycin $50 \mathrm{mg} / \mathrm{L}$ and rifampicin $50 \mathrm{mg} / \mathrm{L}$ ). A single colony was analyzed by PCR using $\mathrm{P} 1$ and $\mathrm{P} 2$ primers, inoculated into $50 \mathrm{~mL}$ of YEB medium containing kanamycin $(50 \mathrm{mg} / \mathrm{L})$ and rifampicin $50 \mathrm{mg} / \mathrm{L}$, and grown at $28^{\circ} \mathrm{C}, 240 \mathrm{rpm}$ until the $\mathrm{OD}_{600}$ reached 1.0. The culture was pelleted by centrifugation and re-suspended in MS liquid medium to obtain an approximate $\mathrm{OD}_{600}$ of 0.5 . Explants $(0.5 \mathrm{~cm} \times 0.5 \mathrm{~cm})$ were excised from 2- to 3-week-old sterile $S$. miltiorrhiza seedlings and immersed for 5 to $8 \mathrm{~min}$ in the Agrobacterium suspension described above for 5 to $8 \mathrm{~min}$. The explants were then blotted on sterile filter paper and plated on a co-cultivation medium (MS, 6-BA $2.0 \mathrm{mg} / \mathrm{L}$ ) in the dark for 2 days at $25^{\circ} \mathrm{C}$. After co-culture, the explants were transferred onto selection medium (MS, 6-BA $1.0 \mathrm{mg} / \mathrm{L}$, kanamycin $50 \mathrm{mg} / \mathrm{L}$, cefotaxime $400 \mathrm{mg} / \mathrm{L}$ ). Cultures were incubated at $25^{\circ} \mathrm{C} / 23^{\circ} \mathrm{C}$ (day/night temperature) with a 16-hr photoperiod. Explants were transferred to fresh selection medium every 2 weeks to generate shoots. Shoots were then transferred to a rooting medium $(1 / 2 \mathrm{MS}$, sucrose $2.0 \%$, kanamycin $30 \mathrm{mg} / \mathrm{L}$ ) to obtain roots. Plants were acclimated for two weeks in vermiculite with half strength MS liquid medium and then transferred to soil.

\section{Southern blot analysis of putative transgenic plants}

Putative transgenic plants were analyzed by DNA hybridization. Total leaf genomic DNA was extracted according to Minas et al. [34]. Thirty $\mu \mathrm{g}$ DNA was digested overnight with single site EcoR I or Hind III restriction enzymes. The digested product was run on a $0.8 \%$ agarose gel. Gel denaturation and neutralization was followed by a routine DNA Southern Blot [35]. The gel was blotted on positively charged nylon membranes by capillary transfer with $20 \times$ SSC following the protocol described in the instruction manual for the DIG High Prime DNA labeling and detection kit (Roche Diagnostics, Indianapolis, USA). DNA fixation, hybridization and wash procedures also followed the manufacturer's instructions. DIG-labeled probe was amplified with $f g f-1$ specific primers $\mathrm{P} 1$ and $\mathrm{P} 4$. Immunological detection was performed according to the manufacturer's instructions. 


\section{ELISA and immunoblot analysis}

The ELISA procedure was performed according to the method described in the instruction manual of the Human FGF-acidic ELISA Construction Kit (Antigenix America, Huntington Station, USA). Standard FGF-1 produced in E.coli (Wanxing-Bio, Shanghai, China) was used to create a series of dilutions $(0,3.125,6.25,12.5,25,50$ and $100 \mathrm{ng} / \mathrm{mL}$ ) in 1XPBS (phosphate buffered saline) buffer to construct a standard curve. Total soluble protein extracts were made by grinding $20 \mathrm{mg}$ of fresh leaf material in $200 \mu \mathrm{L} 1 \mathrm{XPBS}$ buffer. The mixture was centrifuged at $12,000 \mathrm{~g}$ for $10 \mathrm{~min}$ at $4^{\circ} \mathrm{C}$. Recombinant FGF-1 (rFGF-1) protein present in $100 \mu \mathrm{L}$ supernatant was loaded in duplicate wells of a microtiter plate and the $\mathrm{OD}_{650}$ was obtained. The concentration of rFGF-1 was determined by comparison with the constructed FGF-1 standard curve.

For immunoblot analysis, $50 \mathrm{mg}$ of leaf disks were ground in $200 \mu \mathrm{L}$ of deionizer water. The extract was centrifuged at $12,000 \mathrm{~g}$ for $10 \mathrm{~min}$ at $4^{\circ} \mathrm{C} .100 \mu \mathrm{L}$ of supernatant was collected and concentrated to $20 \mu \mathrm{L}$ at $4{ }^{\circ} \mathrm{C}$ for 5 min using a vacuum concentrator (SPD1010 SpeedVac Syetem, Thermo Scientific, USA). $10 \mu \mathrm{L}$ of the concentrated supernatant was separated by $15 \%$ SDS-PAGE, and transferred to a nitrocellulose membrane with an iBlot ${ }^{\circ}$ blotting system (Invitrogen). Western blot analysis of rFGF-1 was performed as described by Li et al. (2012). FGF-1 antibody (Sino Biological Inc. Beijing, China) was used as the primary antibody. Nonspecific binding was blocked by incubation in 3\% bovine serum albumin (BSA) in TBST (25 mM Tris- $\mathrm{HCl}, \mathrm{pH} 7.4,0.14 \mathrm{mM}$ $\mathrm{NaCl}$, and $0.05 \%$ Tween 20) for $2 \mathrm{~h}$ at $4^{\circ} \mathrm{C}$. The membrane was washed three times for $10 \mathrm{~min}$ each in TBST and then incubated in streptavidin-HRP for $1 \mathrm{~h}$. The membrane was then wash three times in TBST and then stained with 4-Chloro-1-naphthol (4CN) to visualize the results.

\section{Cellular proliferation}

The effect of wild-type S. miltiorrhiza (WT), transgenic S. miltiorrhiza (T-SM) or standard FGF-1 (S-FGF) on cell viability and proliferation was examined by thiazolyl blue tetrazolium bromide (MTT) assay described by Mosman [36]. Leaf tissue (0.25 $\mathrm{g}$ fresh weight) of WT and T-SM was ground in $0.75 \mathrm{~mL}$ of $1 \mathrm{XPBS}$ buffer respectively, and centrifuged at $12,000 \mathrm{~g}$ for $10 \mathrm{~min}$ at $4^{\circ} \mathrm{C}$. The supernatants were diluted 5 times in RPMI 1640 medium supplemented with $0.4 \%$ fetal bovine serum (FBS). The yield of rFGF-1 in diluted aqueous extract from T-SM was $10 \mathrm{ng} / \mathrm{mL}$ determined by ELISA assay. S-FGF ( $200 \mathrm{ng} / \mathrm{mL}$, enzyme activity unit: $50 \mathrm{AU} / \mathrm{mL}$ ) was used as positive control. RPMI 1640 medium with $0.4 \%$ FBS was used as blank control. All samples (WT, S-FGF and S-FGF) were 4-fold serially ( $4^{\circ}$ - to $4^{5}$ - fold dilution) diluted in RPMI 1640 medium with $0.4 \%$ FBS.
A concentration of $6.5 \times 10^{3} \mathrm{BALB} / \mathrm{c} 3 \mathrm{~T} 3$ cells per well were seeded in a 96-well microplate containing $100 \mu \mathrm{L}$ of RPMI 1640 medium with $10 \%$ FBS. After $24 \mathrm{~h}$ of incubation in a humidified incubator with $5 \% \mathrm{CO}_{2}$ at $37^{\circ} \mathrm{C}$, all the medium was refreshed in $100 \mu \mathrm{L}$ of RPMI 1640 with $0.4 \%$ FBS. The cultures were maintained for another $24 \mathrm{~h}$. And then, the cells were exposed to $100 \mu \mathrm{L}$ of sample solutions prepared previously with $100 \mathrm{U} / \mathrm{mL}$ penicillin and $100 \mu \mathrm{g} / \mathrm{mL}$ streptomycin. After $72 \mathrm{~h}$ incubation, $25 \mu \mathrm{L}$ of $5 \mathrm{mg} / \mathrm{mL}$ MTT (AMRESCO, USA) was added to each well. After $5 \mathrm{~h}$ treatment with MTT, $120 \mu \mathrm{L}$ of $50 \%$ DMSO was added to the well and mixed thoroughly with pipette. The plate was shaken at room temperature $15 \mathrm{~min}$. The absorbance of each well was determined at $570 \mathrm{~nm}$ using a microplate reader (Bio-rad, USA). Four replicates from each group were analyzed. The experiment was repeated 3 times. Results are expressed as mean \pm standard error. Student's $t$ test was used to evaluate significance differences between groups at $p<0.05$.

\section{CAM assay}

The effect of various extracts on angiogenesis (blood vessel growth) was analyzed by a CAM assay using methycellulose disks described by Larger et al. [37]. Each disk was wetted with $5 \mu \mathrm{L}$ of aqueous extract from either non-transgenic, wild-type S. miltiorrhiza (WT) or transgenic S. miltiorrhiza (T-SM), plants. $5 \mu \mathrm{L}$ of a saline solution and $50 \mathrm{ng}$ of standard FGF-1 (S-FGF) were used as negative and positive controls. $10 \mu \mathrm{L}$ of $0.5 \%$ methylcellulose was dried in a well, $2 \mathrm{~mm}$ diameter and $0.6 \mathrm{~mm}$ deep, of culture plate. Forty five chick eggs were cleaned with a formalin solution and then incubated at $37^{\circ} \mathrm{C}$ and $75 \% \mathrm{RH}$. Abnormal embryo development was verified and the embryos with malformations or dead embryos were discarded. On day 9 of the incubation period, previously prepared saline, S-FGF, T-SM, or WT methylcellulose disks were placed on the CAM. The treated area was carefully excised after $48 \mathrm{~h}$ of incubation and washed with deionized water. The excised area was spread on 3MM filter paper and photographed with a Sony DSLR digital camera.

\section{Burn wound surface area assay on SD rats}

Male SD rats were anesthetized using diethyl ether. Their dorsal hair was shaved as clean as possible and the skin sterilized using $70 \%$ ethanol. A $9-\mathrm{cm}$ by $8-\mathrm{cm}$ square of dorsal skin was further depilated using $8 \%$ sodium sulfide. The bare skin was sterilized using $1 \%$ povidone iodine solution and cleaned with $0.9 \% \mathrm{NaCl}$. Four circular $\left(2.5 \mathrm{~cm}^{2}\right)$ second degree burns were produced with YLS-5Q (Biowill Co, LTD. Shanghai, China) on the dorsal skin of each SD rat. The burn device was set at $80^{\circ} \mathrm{C}$ and applied for 8 seconds. After surgery, each wound was treated for $5 \mathrm{~min}$ with either $100 \mu \mathrm{L}$ of saline, S-FGF 
(100 ng FGF-1), or $100 \mu \mathrm{L}$ of T-SM or WT saline aqueous extract for $5 \mathrm{~min}$. The wound was then covered with sterile gauze. After treatment, each rat was housed in an individual cage, $16 \times 16 \times 9 \mathrm{~cm}$. The burn area was treated once for 5 minutes every other day for 21 days. During the experimental period, all the rats had free access to fresh water and pelleted feed. The wound surface area was determined using the transparent tracing method described by Bohannon and Pfaller [38]. Wound area $=$ $(7.5 \mathrm{~cm} \times 7.5 \mathrm{~cm} \times$ paper weight $) / 0.1604 \mathrm{~g}$. The area of each wound was recorded on day $3(\mathrm{n}=15), 7(\mathrm{n}=15), 14$ $(n=12)$ and $21(n=9)$. Results are expressed as mean \pm standard error. Student's $t$ test was used to evaluate significance differences between groups at $\mathrm{p}<0.05$.

\section{Histological staining}

Two rats were sacrificed on the day or one day after the burn was administered to determine the severity of the burn. Three rats were euthanized after 7-, 14-, and $21 \mathrm{~d}$ of treatment with the different solutions (Saline, S-FGF, T-SM and WT). Tissues including and surrounding the burn wounds were removed and frozen in liquid nitrogen. Wound tissues were cut and processed into $2-\mu \mathrm{m}$ thick cryocut sections. The effect of different treatments on the wound healing process were determined histologically using a hematoxylin $(\mathrm{H})$ and eosin $(\mathrm{E})$ staining kit (Huaxia Bio Co., Beijing, China). The status of the wound was determined by the presence of inflammatory cells, necrotic cells and tissue, epidermal appendages, granulation tissue, and angiogenesis. Images were taken in the center of each histological section at a $200 \times$ magnification.

\section{Additional files}

Additional file 1: Table S1. Analysis of recombinant FGF-1 accumulation in the selected transgenic S. miltiorrhiza lines. Recombinant FGF-1 accumulation levels in aqueous extract from transgenic lines were analyzed by ELISA. The yield was calculated by the standard FGF-1 curve $(Y=0.0042 X$ $+0.1418)$. $Y$ is the protein measurement of $O_{650}$ in $100 \mu \mathrm{L}$ of 1 XPBS buffer. FW: fresh weight of the leaf. The detailed method was described in material and method. Data represents mean of duplicates.

Additional file 2: Figure S1. Southern blot. DNA hybrization assay using $f g f-1$ as a probe. Genomic DNA $(30 \mu \mathrm{g})$ from wild-type (WT) and the transgenic (T117) plants was digested with either, HindIII or EcoRl restriction enzymes. Lane 1, wild type. Lane 2-9, transgenic lines. Lane 1, fgf-1 PCR product; Lane 2, WT/Hindlli; Lane 3, WT/EcoRl, Lane 4, T117/HindIII; Lane 5, T117/EcoRl.

Additional file 3: Figure S2. Deep-second burn degree determined by H\&E analysis. Normal skin had an intact cuticle, hair follicles, sebaceous glands, collagen and a prickle cell layer (A and B). Epidermal necrolysis was observed $24 \mathrm{~h}$ after burn treatment ( $C$ and $D)$. Micrographs were taken at $200 x$

\section{Abbreviations}

FGF: Fibroblast growth factor; CAM: Chicken embryo chorioallantoic membrane assay; MTT: Thiazolyl blue tetrazolium bromide colorimetric assay; TCM: Traditional Chinese medicine.

\section{Competing interests}

The authors declare that they have no competing interests.

\section{Authors' contributions}

$Y T$ carried out the gene cloning, participated in the plant transformation, experiments and drafted the manuscript. KW designed and analyzed data, and helped to write the paper. NW and GL participated in experiments. DL conceived of the study, and participated in its design and coordination and helped to draft the manuscript. All authors read and approved the final manuscript.

\section{Acknowledgements}

This work was supported by the Chinese National Biotechnology Development Plan (2013ZX08005-004), The Researcher Foundation of the Chinese Academy of Agricultural Sciences, and the National Institute of General Medical Sciences of the National Institutes of Health (8P20GM103447).

\section{Author details}

${ }^{1}$ Biotechnology Research Institute, Chinese Academy of Agricultural Sciences, 12 Southern Zhong Guan Cun Road, Beijing 100081, China. ${ }^{2}$ Department of Natural Sciences, Northeastern State University, Broken Arrow, Oklahoma 74014, USA

Received: 10 June 2014 Accepted: 5 August 2014

Published: 9 August 2014

\section{References}

1. Peck MD: Epidemiology of burns throughout the world. Part I: distribution and risk factors. Burns 2011, 37:1087-1100. doi:10.1016/j.burns.2011.06.005.

2. National Institute of General Medical Sciences. http://www.nigms.nih.gov/ Education/Factsheet_Burns.htm.

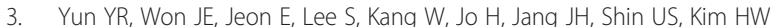
Fibroblast growth factors: biology, function, and application for tissue regeneration. J Tiss Eng 2010, 148142:. doi:10.4061/2010/218142.

4. Zakrzewska M, Marcinkowska E, Wiedlocha A: FGF-1: from biology through engineering to potential medical applications. Crit Rev Clin Lab Sci 2008, 45:91-135. doi:10.1080/10408360701713120.

5. Zazo M, Lozano RM, Ortega S, Varela J, Díaz-Orejas R, Ramírez JM, Giménez-Gallego G: High-level synthesis in Escherichia coli of shortened and full-length human acidic fibroblast growth factor and purification in a form stable in aqueous solutions. Gene 1992, 113:231-238.

6. Fantoni A, Bill RM, Gustafsson L, Hedfalk K: Improved yields of full-length functional human FGF1 can be achieved using the methylotrophic yeast Pichia pastoris. Protein Expr Purif 2007, 52:31-39.

7. Zhou Y, Ren LZ, Zhu JG, Yan S, Wang H, Song N, Li L, Ouyang H, Pang D: Construction of a recombinant human FGF1 expression vector for mammary gland-specific expression in human breast cancer cells. Mol Cell Biochem 2011, 354:39-46.

8. Liu J, Ma P, Sun Y, Yang M, Li Y, Wu Y, Zhu X, Wang X: Expression of human acidic fibroblast growth factor in Nicotiana benthamiana with a potato-virus-X-based binary vector. Biotechnol Appl Biochem 2007, 48:143-147.

9. Pandit AS, Wilson DJ, Feldman DS: Fibrin scaffold as an effective vehicle for the delivery of acidic fibroblast growth factor (FGF-1). Biomater Appl 2000, 14:229-242

10. Tan Y, Xiao J, Huang Z, Xiao Y, Lin S, Jin L, Feng W, Cai L, Li X: Comparison of the therapeutic effects recombinant human acidic and basic fibroblast growth factors in wound healing in diabetic patients. $J$ Health Sci 2008, 54:432-440. doi:10.1248/jhs.54.432.

11. Wang B-Q: Salvia miltiorrhiza: chemical and pharmacological review of a medicinal plant. J Med Plants Res 2010, 4:2813-2820.

12. Yin HQ, Choi YJ, Kim YC, Sohn DH, Ryu SY, Lee BH: Salvia miltiorrhiza Bunge and its active component cryptotanshinone protects primary cultured rat hepatocytes from acute ethanol-induced cytotoxicity and fatty infiltration. Food Chem Toxicol 2009, 47:98-103.

13. Liu CS, Cheng Y, Hu JF, Zhang W, Chen NH, Zhang JT: Comparison of antioxidant activities between salvianolic acid B and Ginkgo biloba extract (EGb 761). Acta Pharmacol Sin 2006, 27:1137-1145. 
14. Zhou ZT, Yang Y, Ge JP: The preventive effect of salvianolic acid B on malignant transformation of DMBA-induced oral premalignant lesion in hamsters. Carcinogenesis 2006, 27:826-832.

15. Park JW, Lee SH, Yang MK, Lee JJ, Song MJ, Ryu SY, Chung HJ, Won HS, Lee CS, Kwon SH, Yun YP, Choi WS, Shin HS: 15,16-Dihydrotanshinone I, a major component from Salvia miltiorrhiza Bunge (Dansham), inhibits rabbit platelet aggregation by suppressing intracellular calcium mobilization. Arch Pharm Res 2008, 31:47-53.

16. De Jaeger G, Scheffer S, Jacobs A, Zambre M, Zobell O, Goossens A, Depicker A, Angenon G: Boosting heterologous protein production in transgenic dicotyledonous seeds using Phaseolus vulgaris regulatory sequences. Nature Biotechnol 2002, 20:1265-1268.

17. Hood EE, Woodard SL, Horn ME: Antibody manufacturing in transgenic plants: myths and realities. Curr Opin Biotechnol 2002, 13:630-635.

18. Lay IS, Chiu JH, Shiao MS, Liu WY, Wu CW: Crude extract of Salvia miltiorrhiza and salvianolic acid B enhance in vitro angiogenesis in murine SVR endothelial cell line. Planta Med 2003, 69:26-32.

19. Ding LT, Zhu YG, Gu ZQ, Cai LL, Peng JL, Lü GZ: Effect of Salvia miltiorrhiza and Ligustrazine injection on the early myocardial damage of patients with severe burn. Zhonghua Shao Shang Za Zhi (in Chinese) 2012 28:170-172.

20. Chen J, Lv Q, Yu M, Zhang X, Gou J: Randomized clinical trial of Chinese herbal medications to reduce wound complications after mastectomy for breast carcinoma. Br J Surg 2010, 97:1798-1804.

21. Yin $X$, Yin Y, Cao FL, Chen YF, Peng Y, Hou W-G, Sun SK, Luo ZJ: Tanshinone IIA attenuates the inflammatory response and apoptosis after traumatic injury of the spinal cord in adult rats. PLoS One 2012, 7(6):e38381. doi:10.1371/journal.pone.0038381

22. Chen YS, Lee SM, Lin YJ, Chiang SH, Lin CC: Effects of danshensu and salvianolic Acid B from Salvia miltiorrhiza Bunge (Lamiaceae) on cell proliferation and collagen and melanin production. Molecules 2014, 19:2029-2041. doi:10.3390/molecules19022029.

23. Pan RH, Xie FY, Chen HM, Xu LZ, Wu XC, Xu LL, Yao G: Salvianolic acid B reverses the epithelial-to-mesenchymal transition of HK-2 cells that is induced by transforming growth factor- $\beta$. Arch Pharm Res 2011, 34:477-483.

24. Caron A, Michelet $S$, Caron A, Sordello S, Ivanov MA, Delaère P, Branellec D, Schwartz B, Emmanuel F: Human FGF-1 gene transfer promotes the formation of collateral vessels and arterioles in ischemic muscles of hypercholesterolemic hamsters. J Gene Med 2004, 6:1033-1045.

25. Cao RH, Brakenhielm E, Pawliuk R, Wariaro D, Post MJ, Wahlberg E, Leboulch P, Cao $Y$ : Angiogenic synergism, vascular stability and improvement of hind-limb ischemia by a combination of PDGF-BB and FGF-2. Nature Med 2003, 9:604-613.

26. Faye L, Gomord V: Post translational modification of therapeutic proteins in plants. Curr Opin Plant Biol 2004, 7:171-181.

27. Feng $H$, Xiang $H$, Zhang J, Liu G, Guo N, Wang X, Wu X, Deng X, Yu L: Genome-wide transcriptional profiling of the response of Staphylococcus aureus to cryptotanshinone. J Biomed Biotechnol 2009, 2009:617509. doi:10.1155/2009/617509.

28. Andreopoulos FM, Persaud I: Delivery of basic fibroblast growth factor (bFGF) from photoresponsive hydrogel scaffolds. Biomaterials 2006, 27:2468-2476

29. Cai S, Liu Y, Xiao ZS, Prestwich GD: Injectable glycosaminoglycan hydrogels for controlled release of human basic fibroblast growth factor. Biomaterials 2005, 26:6054-6067.

30. Bardor M, Faveeuw C, Fitchette A-C, Gilbert D, Galas L, Trottein F, Faye L, Lerouge P: Immunoreactivity in mammals of two typical plant glyco-epitopes, core a(1,3)-fucose and core xylose. Glycobiology 2003, 13:427-434.

31. During K, Hippe S, Kreuzaler F, Schell J: Synthesis and self-assembly of a functional monoclonal antibody in transgenic Nicotiana tabacum. Plant Mol Biol 1990, 15:281-293.

32. Chen MH, Huang LF, Li HM, Chen YR, Yu SM: Signal peptide-dependent targeting of a rice a-amylase and cargo proteins to plastids and extracellular compartments of plant cells. Plant Physiol 2004, 135:1367-1377.

33. Horsch RB, Fry JE, Hofimann NL, Eichholtz D, Rogers SG, Fraley RT: A simple and general method for transferring genes into plants. Science 1985, 227:1229-1231.
34. Minas K, McEwan NR, Newbold CJ, Scott KP: Optimization of a high-throughput CTAB-based protocol for the extraction of qPCR-grade DNA from rumen fluid, plant and bacterial pure cultures. FEMS Microbiol Lett 2011, 25:162-169. doi:10.1111/j.1574-6968.2011.02424x

35. Li G, Wang KY, Li D, Wang N, Liu D: Cloning, expression and characterization of a gene from earthworm Eisenia foetida encoding a blood-clot dissolving protein. PLoS One 2012, 7:e53110. doi:10.1371/journal.pone.0053110.

36. Mosmann T: Rapid colorimetric assay for cellular growth and survival: application to proliferation and cytotoxicity assays. J Immunol Methods 1983, 65:55-63.

37. Larger E, Marre M, Corvol P, Gasc JM: Hyperglycemia-induced defects in angiogenesis in the chicken chorioallantoic membrane model. Diabetes 2004, 53:752-761.

38. Bohannon RW, Pfaller BA: Documentation of wound surface area from tracings of wound perimeters. Pbys Tkr 1983, 63:1622-1624.

doi:10.1186/1472-6750-14-74

Cite this article as: Tan et al:: Ectopic expression of human acidic fibroblast growth factor 1 in the medicinal plant, Salvia miltiorrhiza, accelerates the healing of burn wounds. BMC Biotechnology 2014 14:74.

\section{Submit your next manuscript to BioMed Central and take full advantage of:}

- Convenient online submission

- Thorough peer review

- No space constraints or color figure charges

- Immediate publication on acceptance

- Inclusion in PubMed, CAS, Scopus and Google Scholar

- Research which is freely available for redistribution

Submit your manuscript at www.biomedcentral.com/submit
C Biomed Central 